\title{
Using Virtual Reality (VR) for Real-Time Tomography Exploration of Nanoparticles
}

\author{
Aaron Klick ${ }^{1}$, Jacob Brink ${ }^{2}$ and M.J. Kim ${ }^{1,3}$ \\ 1. School of Art, Technology and Emerging Communication, University of Texas at Dallas, Richardson, \\ TX. \\ 2. JEOL USA, Inc., Peabody, MA. \\ 3. Department of Materials Science and Engineering, University of Texas at Dallas, Richardson, TX.
}

Currently, image based tomography uses simple desktop applications for viewing. What if this could be transferred and viewed in real time through VR? That was the main question that we asked ourselves. We tested this with a set of bimetallic Pd-Pt nanocrystal tomographic images [1]. The primary goal was to make it function on mobile device units such as the GearVR.

Each image size in the set is $511 \times 707$ and with 100 image layers. This is a combined total of $36,127,700$ points of data. That amount of data is too much for current mobile devices. So, to eliminate unneeded background noise in each image, two image processing techniques were used. The first technique is a simple value thresholding, where in we only accept points of data in a specific value range as valid. The second technique used is a spatial threshold. The spatial threshold examines each image to see if each point of data is surrounded by at least 12 other valid points of data, after the value threshold [2]. Using both techniques on the image set allowed us to reduce the point count to roughly 2.8 million - while still maintaining the structure of the nanoparticles as close as possible. The reduced point count easily allows it to work on current Samsung Galaxy S8 phones and also prior Samsung Galaxy S7 phones with GearVR. Fig. 1 demonstrates the difference between raw data and a simple value threshold applied. The concave features of about $1 \mathrm{~nm}$ deep on the nanoparticle surfaces are clearly resolved in the threshold applied images.

Nonetheless, one last issue needed to be addressed for a usable experience. Since the points are aligned in a column and row fashion from an image, it suffers from a moiré pattern when viewed up close. To address this issue, while maintaining the structure, each point of data was offset visually by a random value of 0.5 to +0.5 in position in $3 \mathrm{D}$ space. This still maintains the structure of the data, while removing the moiré pattern. As shown in Fig. 2, the VR ready data set retains the integrity of the core-frame concave structure of Pd-Pt bimetallic nanoparticles.

Even though, we aimed for GearVR with the Samsung S7 and S8 devices, the transformed data set can also work on an iPhone 7 and above. Of course, the experience is not limited to just mobile devices. It can also be used with the Oculus or Vive on desktops [3].

\section{References:}

[1] Ning Lu et al, J. Phys. Chem. C 118 (2014), p. 28876.

[2] Gonzalez, Rafael C. \& Woods, Richard E. in "In Digital Image Processing”, (Pearson Education) p. 595.

[3] This work was supported in part by Louis Beecherl, Jr. endowment funds. 
(a)

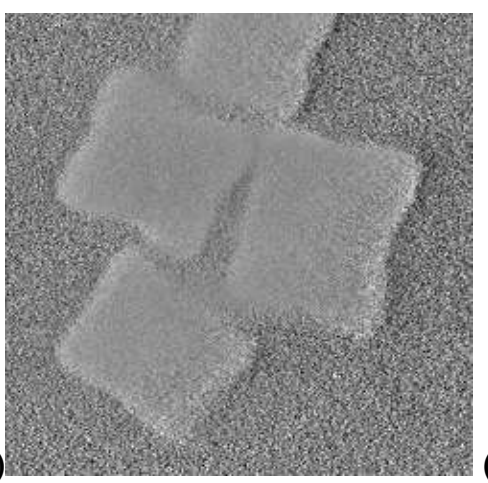

(b)

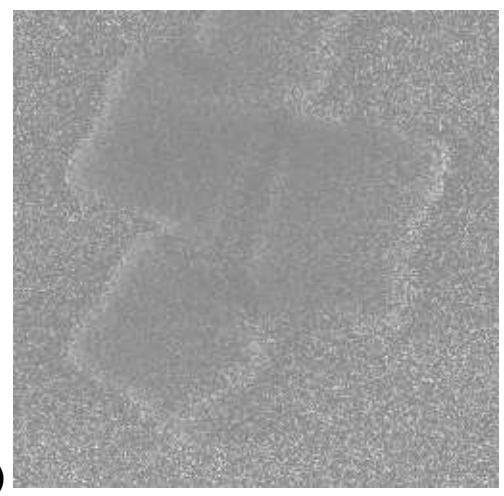

Figure 1. (a) Small section of raw image data. (b) Small section of image data with value threshold applied for all 100 layers. Full data size is 12.5 million points of data. Each nanoparticle is about $20 \mathrm{~nm}$ in size. About $1 \mathrm{~nm}$ concave features are retained even with the threshold value applied.

(a)

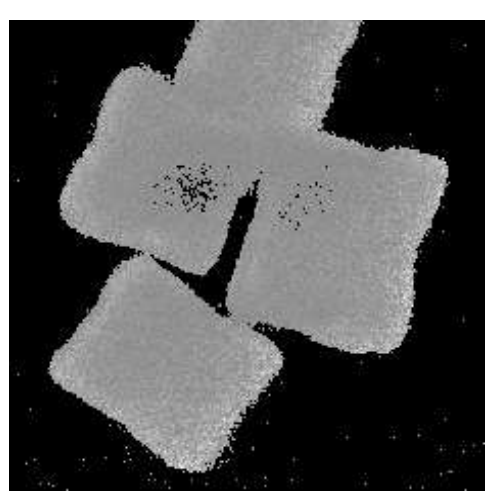

(b)

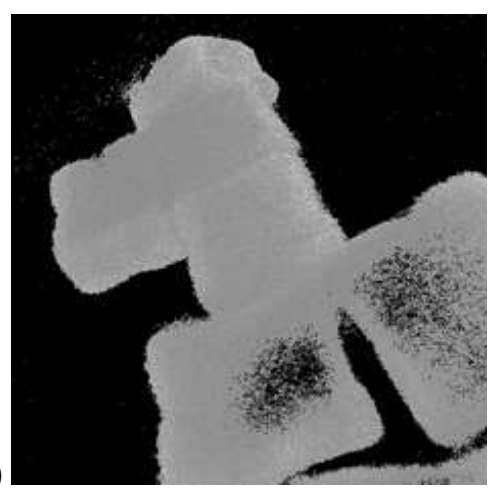

Figure 2. (a) Small section of data with both value threshold and spatial threshold applied. Full data size is 2.8 million points. (b) Another view of value plus spatial threshold applied.

(a)
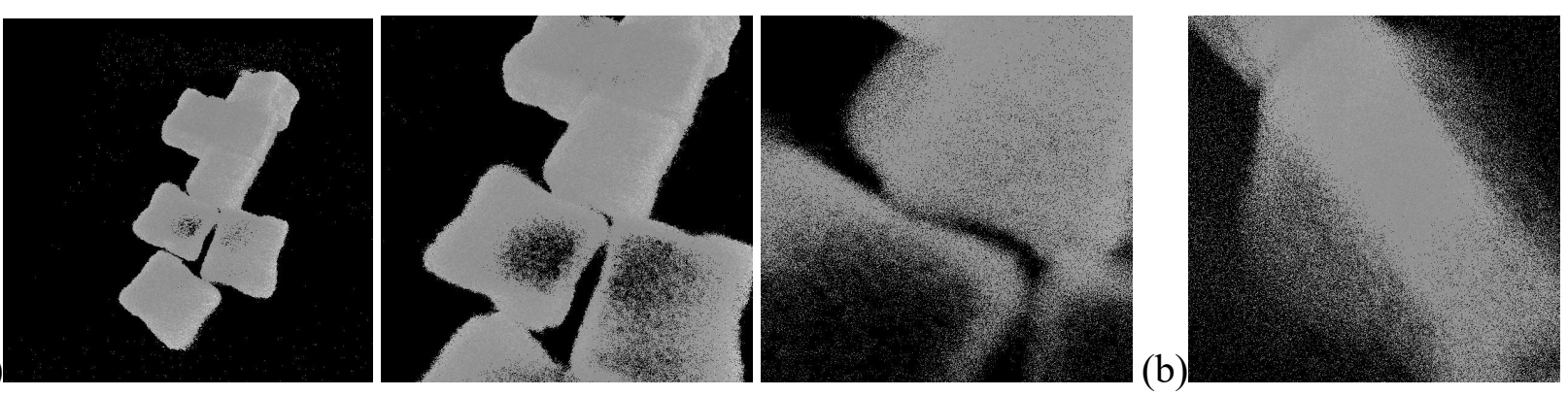

Figure 3. (a) Moving from far away to the inside of one of the nanoparticles (b). 